\title{
Phase transition in conservative diffusive contact processes
}

\author{
Carlos E. Fiore and Mário J. de Oliveira \\ Instituto de Física \\ Universidade de São Paulo \\ Caixa Postal 66318 \\ 05315-970 São Paulo, São Paulo, Brazil
}

(Dated: October 30, 2018)

\begin{abstract}
We determine the phase diagrams of conservative diffusive contact processes by means of numerical simulations. These models are versions of the ordinary diffusive single-creation, pair-creation and triplet-creation contact processes in which the particle number is conserved. The transition between the frozen and active states was determined by studying the system in the subcritical regime and the nature of the transition, whether continuous or first order, was determined by looking at the fractal dimension of the critical cluster. For the single-creation model the transition remains continuous for any diffusion rate. For pair- and triplet-creation models, however, the transition becomes first order for high enough diffusion rate. Our results indicate that in the limit of infinite diffusion rate the jump in density equals $2 / 3$ for the pair-creation model and $5 / 6$ for the triplet-creation model.
\end{abstract}

PACS numbers: 05.50.Ln, 05.50+q, 05.65.+b 


\section{INTRODUCTION}

The usual contact process [1, 2, 3] is a model for nonequilibrium process composed by two subprocesses: a catalytic creation and a spontaneous annihilation. In the usual contact process, which we call single-creation contact process, a particle is created if the site has at least one neighbor site occupied. Many generalizations [4, 5, 6] can be made by taking into account the basic mechanisms contained in the single-creation model. In the pair-creation contact process, for instance, at least two neighbor sites occupied are necessary to create a new particle. In the triplet-creation model one should have at least three sites occupied. All these variants of the contact process exhibit a continuous phase transition between an absorbing state and an active state that belongs to the direct percolation (DP) universality class.

Diffusive models are defined by permitting the diffusion of particles in addition to the catalytic creation and spontaneous annihilation. A diffusion process is done by moving a particle to an empty nearest neighbor site. The introduction of diffusion does not destroy the transition from an active state to an absorbing state giving rise to a transition line that separates the two phases. Jensen and Dickman [7] have shown that for the single-creation diffusive contact process this line is always second order for any diffusion rate and belongs to the DP universality class, i. e., the addition of the diffusion does not change the universality class nor the nature of transition. For the pair-creation the numerical results by Dickman and Tomé [8] show that the transition is continuous for low diffusion and they argue that there is no change in the nature of the transition for high diffusion. For the triplet-creation contact process, Dickman and Tomé [8] have shown that the transition becomes a first order for high enough diffusion.

In the present work, we study conservative versions of the models mentioned above. A conservative version of a model for nonequilibrium process was introduced by Ziff and Brozilow [9], who used a constant-density ensemble to study the ZGB model. A conservative version of directed percolation was used by Bröker and Grassberger [10]. The conservative contact process was introduced by Tomé and de Oliveira [11] who have shown its equivalence in the thermodynamic limit to the ordinary contact process and how to calculate the rates from averages over the constant-density ensemble. The equivalence between this ensemble and the constant-rate ensemble was later proved by Hilhorst and Wijland [12]. 
In the conservative versions of contact processes [11, 13, 14], an empty site becomes occupied in a way similar to the catalytic creation. But instead of creating a new particle, like in the ordinary contact processes, a randomly chosen particle of the system leaves its place and jumps into the empty site. Thus, both the creation and annihilation processes are replaced with a jumping process. However, this is not an unrestricted jumping because particles are not allowed to jump to a vacant site surrounded by empty sites. It is necessary to have a neighborhood (a set of one, two, or three sites, depending on the model) of sites occupied.

One advantage of using the conservative versions is that they allow us to study the model without the danger of falling down into the absorbing state. The conservation of particles permits us to perform numerical simulations that avoid the accidental fall into the absorbing state. Although they do not have absorbing states, they are equivalent, in the thermodynamic limit, to the ordinary models. The conservative and ordinary models are versions of the same model in distinct ensembles [11, 12, 13, 14]; the first models belonging to the constant-particle ensemble, the second models belonging to the constant-rate ensemble.

Another advantage is related to the expected existence of first order transition. In the ordinary models, a very small change in the annihilation rate (the control parameter), near the transition, causes a great change in the density. In the conservative models, because of the fact that the particle number is a conserved quantity (and therefore, works as the control parameter), this problem does not occur. This advantage has been used by Ziff and Brosilow [9] in their study first order transition in the ZGB model.

\section{CONSERVED REACTION DIFFUSION MODELS}

In the construction of conserved models we have to be concerned only with the reaction process since the diffusion process already conserves the particle number. The necessary condition to set up an equivalent conserved version of an arbitrary ordinary reaction-diffusion process in a lattice is that the reaction process be a sum of a creation subprocess and an annihilation subprocess [14]. This is always possible do realize because these two subprocesses are mutually excludent. If a site of the lattice is empty only creation is possible; if it is occupied, only annihilation is possible. Therefore, the transition rate $w_{i}$ related the 
creation-annihilation of a particle at site $i$ can always be written in the form

$$
w_{i}=k_{C} \omega_{i}^{C}+k_{A} \omega_{i}^{A}
$$

where the first term is related to the creation of a particle at site $i$ and the second to annihilation of a particle at site $i$. The quantity $\omega_{i}^{C}$ vanishes if there is already a particle at site $i$ and $\omega_{i}^{A}$ vanishes if site $i$ is empty. The quantities $k_{C}$ and $k_{A}$ are the actual parameters of the ordinary model which we call amplitudes of the creation and annihilation rates, respectively.

The conserved version is set up by replacing both the creation and annihilation subprocesses by a particle jump process $i \rightarrow j$ with rate $w_{i j}=\omega_{i}^{A} \omega_{j}^{C} / L$ where $L$ is the number of sites of the lattice. One can prove [14] that a two site process defined by this transition rate is equivalent in the thermodynamic limit to the ordinary process. To see how this come about let us look at the total rate $\sum_{i} w_{i j}$ in which particles jump to site $j$. In the thermodynamic limit, the sum $\sum_{i} \omega_{i}^{A} / L$ approaches, by the law of large numbers, the average $\left\langle\omega_{i}^{A}\right\rangle$ so that $\sum_{i} w_{i j}=\left\langle\omega_{i}^{A}\right\rangle \omega_{j}^{C}$. By an analogous argument the total rate in which particles leave the site $i$ is $\sum_{j} w_{i j}=\left\langle\omega_{j}^{C}\right\rangle \omega_{i}^{A}$. The averages $\left\langle\omega_{i}^{A}\right\rangle$ and $\left\langle\omega_{i}^{C}\right\rangle$ act then as the amplitudes of creation and annihilation rates, respectively, what allows us to write down the following relation [14]

$$
\frac{k_{A}}{k_{C}}=\frac{\left\langle\omega_{j}^{C}\right\rangle}{\left\langle\omega_{i}^{A}\right\rangle}
$$

between the amplitude rates of the constant-rate ensemble and averages determined in the constant-particle ensemble.

For the model we study here particles are spontaneous annihilated do that $\omega_{i}^{A}$ is 1 if site $i$ is occupied and 0 if it is empty. Therefore $\sum_{i} \omega_{i}^{A}=n$ where $n$ is the number of particles. The quantity $\omega_{j}^{C}$ is 0 if site $j$ is occupied. Since creation is catalytic, this quantity depends also on the neighborhood of site $j$. For the single-creation model it equals half the number of nearest neighbor occupied sites. For the pair-creation model it equals half the number of pairs of nearest neighbor occupied sites. For the triplet-creation model it equals half the number of triplets of nearest neighbor occupied sites. It is convenient do define an active site as the site for which $\omega_{j}^{C}$ is nonzero. The number of active sites $n_{\text {ac }}$ is defined by

$$
n_{\mathrm{ac}}=\sum_{j} w_{j}^{C}
$$


We also define a quantity $\alpha$ as being the right-hand side of equation (2) so that, for the models studied here,

$$
\alpha=\frac{\left\langle n_{\mathrm{ac}}\right\rangle}{n} .
$$

where the averages are taken in the constant-particle ensemble. Usually one defines the ordinary reaction process so that the rate amplitudes are $k_{C}=1$ and $k_{A}=k$. Therefore, according to relation (2), $\alpha$ coincides with the parameter $k$ of the ordinary model as long as the average density of particles of the ordinary models equals the density of particle $n / L$ in the conserved models.

The rules of the reaction-diffusion processes we used are such that the diffusion occurs with probability $D$ and the jump process with probability $1-D$. The quantity $D$ and the diffusion rate $\tilde{D}$ are related by

$$
D=\frac{\tilde{D}}{1+\tilde{D}}
$$

\section{EXACT AND MEAN-FIELD RESULTS}

The average number of active sites per site of the lattice equals the probabilities $P(10)$, $P(110)$, and $P(1110)$ for the single-creation, pair-creation and triplet-creation models, respectively. Since the number o particles per site is the probability $P(1)$ it follows that $\alpha$ is given, respectively, by $\alpha=P(10) / P(1), \alpha=P(110) / P(1)$, and $\alpha=P(1110) / P(1)$, for the

three models. In the limit of infinite diffusion rate the particles will be uncorrelated so that $P(10)=P(1) P(0)$, etc. Taking into account that $P(1)=\rho$ and $P(0)=1-\rho$, we get the following exact results for the active state, valid for $D=1$,

$$
\alpha=1-\rho,
$$

for the single-creation model,

$$
\alpha=\rho(1-\rho),
$$

for the pair-creation model, and

$$
\alpha=\rho^{2}(1-\rho)
$$

for the triplet-creation model.

These results give a continuous transition for the single-creation model. For the paircreation and triplet-creation models, on the other hand, they give a discontinuous transition 
since $\rho$ does not vanishes continuously as one increases $\alpha$. The quantity $\alpha$ has a maximum at a nonzero value $\rho_{0}$ of the density which is $\rho_{0}=1 / 2$ for the pair-creation model and $\rho_{0}=2 / 3$ for the triplet-creation. The corresponding values of $\alpha$ are $\alpha_{0}=1 / 4$ and $\alpha_{0}=4 / 27$, respectively. Since there is no free energy from which we could decide at what point the jump in the density occurs one is tempted to use the maximum value of $\alpha$ (spinodal point). However, as we will see, our numerical results do not support this point of view. According to the numerical results, the discontinuity occurs at a smaller value of $\alpha$.

For the single creation the diffusion does not change the nature of the transition. Even at infinite diffusion rate the transition is continuous as the exact result (6) shows. The critical line on the diagram $D$ versus $\alpha$ can be obtained by a mean-field approximation. By using a two-site mean-field approximation we get a relation between $\rho$ and $\alpha$ which shows a continuous transition for all values of $D$ and which recovers the exact result (6] $)$ when $D=1$. The critical line obtained from this approximation is given by

$$
D=\frac{2 \alpha-1}{4 \alpha-2 \alpha^{2}-1}
$$

showing that $\alpha \rightarrow 1$ as $D \rightarrow 1$ in accordance with the exact result (6) and, as we will see, with numerical simulations.

The exact results (7) and (86) for the pair-creation and triplet-creation models cannot be used to infer that the transition will remain discontinuous for finite diffusion rate even if the rate is large. An indication that the transition is continuous at low diffusion and discontinuous for sufficiently large diffusion, giving rise to a tricritical point, comes from mean-field approximations which can be done at several levels [15]. At the level of three sites the mean-field approximation locates the tricritical point of the pair-creation model at $D_{t}=0.032$ and $\alpha_{t}=0.1687$. For the triplet-creation model it is necessary to use a higher order of approximation. At the level of four sites the tricritical point occurs at $D_{t}=0.017$ [8]. Although both results are in qualitative agreement with our numerical simulations they are very low when compared with the figures coming from the numerical simulations.

\section{NUMERICAL SIMULATIONS}

We have simulated the conservative diffusive contact process in a one-dimensional lattice. The actual simulation is performed as follows. At each time step a particle is selected at 


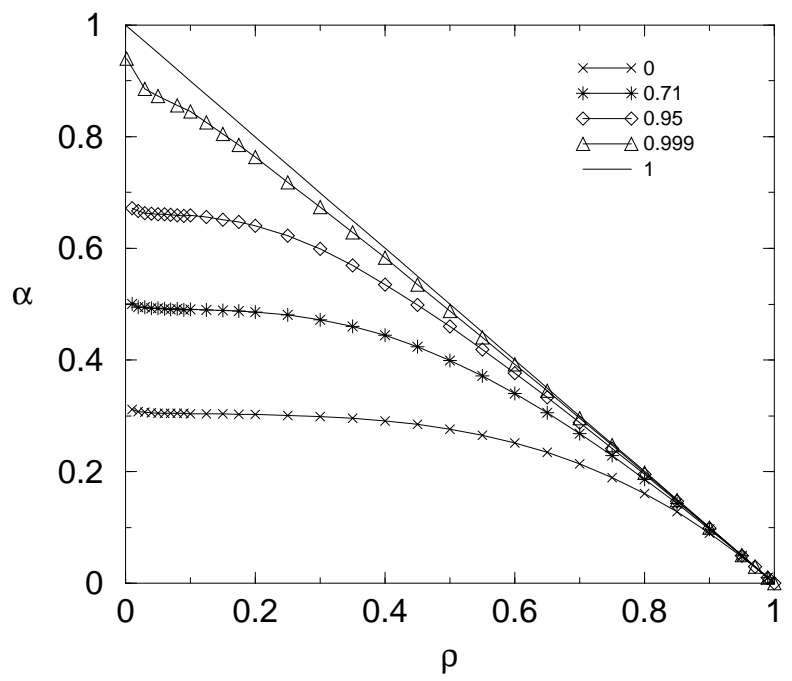

FIG. 1: The effective number of active sites per particle $\alpha$ as function of particle density $\rho$ for single-creation conservative contact process for some values of probability D.

random, say a particle at site $i$, and one of its neighboring sites is chosen randomly, say site $j$. If this neighboring site is empty then we decide which process to perform: the diffusion of particles, occurring with probability $D^{\prime}$, or the creation-annihilation process, occurring with probability $1-D^{\prime}$. If the diffusion process is chosen then the particle at $i$ hops to the neighboring site $j$. If the creation-annihilation process is chosen then any another particle of the system, including the one at site $i$, is chosen randomly and placed at site $j$. In the case of the pair-creation or triplet-creation models, however, this only happens if the chosen particle $i$ has at least one or two nearest neighbor occupied sites, respectively. The relation between the probability $D^{\prime}$ we use in the simulation and the actual probability of diffusion $D$ is $D^{\prime}=2 D /(1+D)[16]$. This is so because we are choosing a particle from a list of occupied sites and then choosing with equal probability one of the neighboring sites to place the particle.

\section{A. Supercritical regime}

The simulation was performed using lattices with $L$ sites and periodic boundary conditions. The averages of the appropriates quantities were obtained from a number of Monte Carlo steps ranging from $10^{6}$ to $3 \times 10^{7}$, after discarding a sufficient number of steps to reach the stationary state. In Figs. 1, 2 and 3, we show the particle density $\rho=n / L$ as a function 


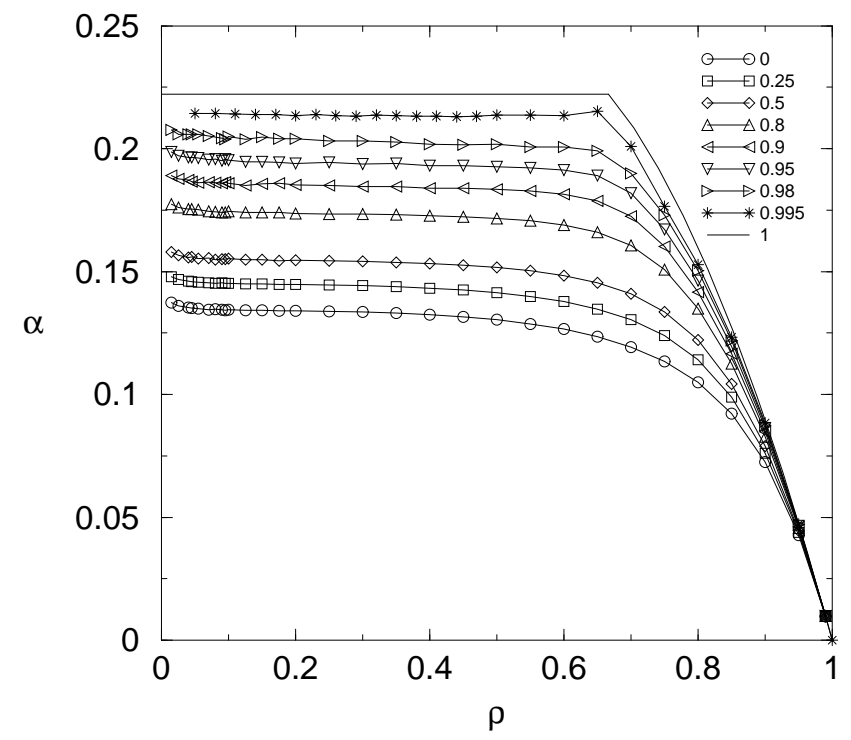

FIG. 2: The effective number of active sites per particle $\alpha$ as function of the particle density $\rho$ for pair-creation conservative contact process for some values of probability $\mathrm{D}$. The horizontal straight line at $\alpha=0.222$ was obtained by extrapolation.

of $\alpha$, calculated by using formula (4), for several values of the hopping probability $D$. We have used $L=10^{4}$ and varied the number of particles $n$. As expected, for high diffusion rate the curves approach the exact behaviors given by equations (6), (7) and (8)).

For the diffusive single-creation contact process, the transition is found to be continuous for all values of $D$. Increasing the diffusion probability $D$ the critical value of $\alpha$ increases towards the value 1 when $D \rightarrow 1$ as expected. For the diffusive pair-creation and tripletcreation models the phase transition is continuous for low diffusion becoming discontinuous for high enough diffusion. The tricritical point occurs at $D_{t}=0.965 \pm 0.010$ for the paircreation contact process and $D_{t}=0.945 \pm 0.005$ for the triplet-creation as we shall see shortly. Figs. 2 and 3 seems to show that this is indeed the case.

To compare the behaviors corresponding to the second and first order transitions, we simulated the pair-creation model at $D=0.5$ and $D=0.995$ for various values of the system size $L$ ranging from 50 to $10^{4}$. For the former case, $D=0.5$, the plot of $\rho$ versus $\alpha$, shown in Fig. 4, shows a continuous transition. That the transition is continuous is confirmed by the the data collapse of the data shown in the inset of Fig. 固. For $D=0.995$, the plot of $\rho$ versus $\alpha$, shown in Fig. 5, displays a jump when $L \rightarrow \infty$ increases. Results similar to those of Figs. 4 and 5 are also found for the triplet-creation model. 


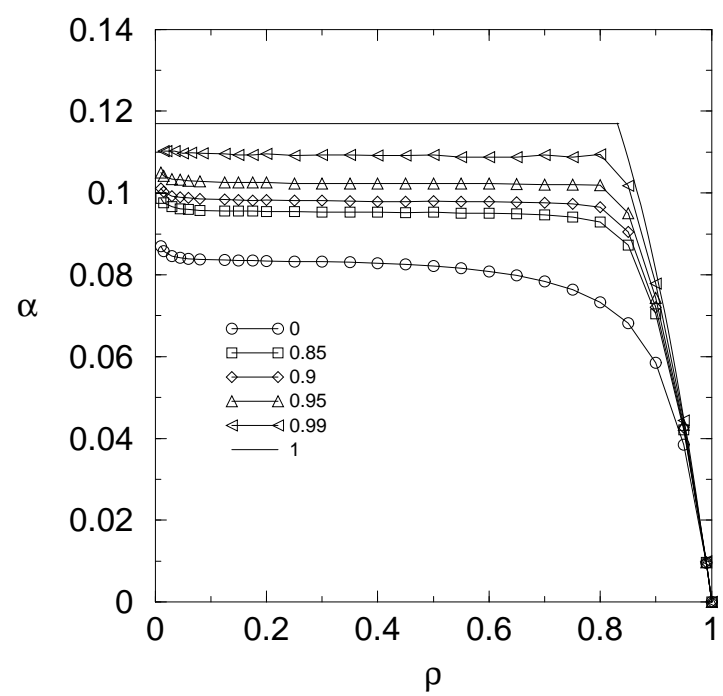

FIG. 3: The effective number of active sites per particle $\alpha$ as function of the particle density $\rho$ for triplet-creation conservative contact process for some values of probability D. The horizontal straight line at $\alpha=0.115$ was obtained by extrapolation.

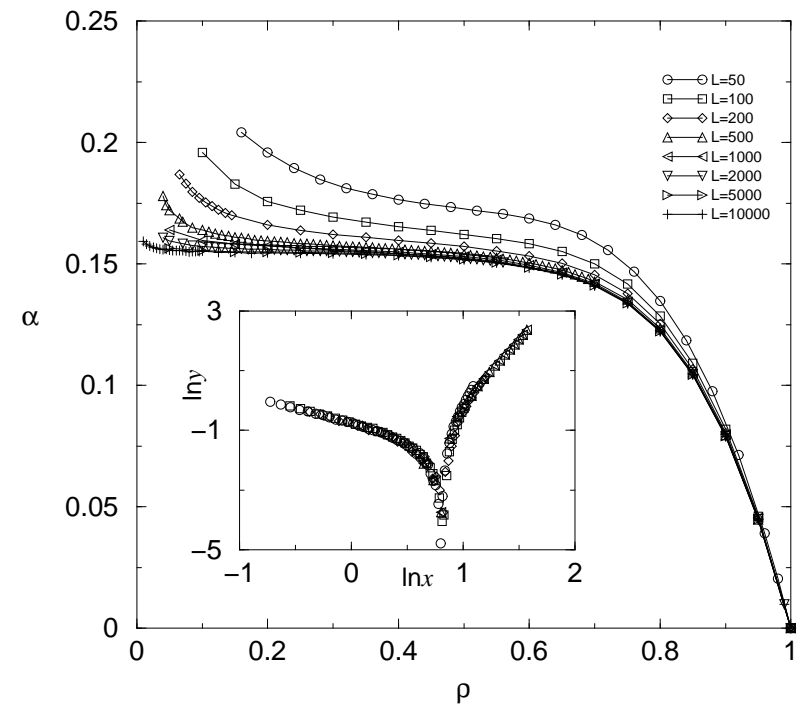

FIG. 4: The effective number of active sites per particle $\alpha$ as function of the particle density $\rho$ for several values of $L$ in the supercritical regime for $D=0.5$ for the pair-creation model. The inset show the scaling plot of $y=L^{\beta / \nu}\left|\alpha_{c}-\alpha\right|$ versus $x=L^{1 / \nu} \rho$, using the DP critical exponents $\beta=0.277$ and $\nu=1.097$. 


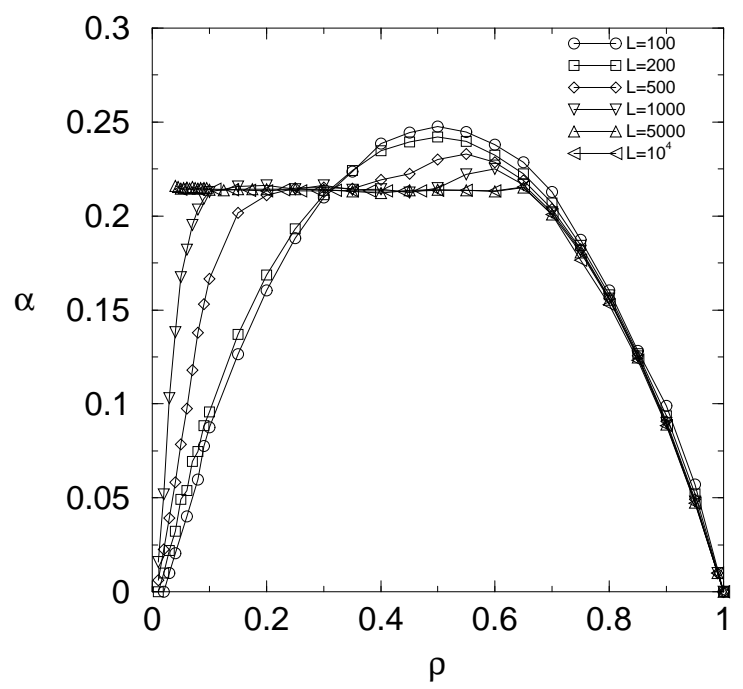

FIG. 5: The effective number of active sites per particle $\alpha$ as function of the particle density $\rho$ for several values of $L$ in the supercritical regime for $D=0.995$ for the pair-creation model.

\section{B. Subcritical regime}

To simulate the system in the subcritical regime we consider an infinite lattice with a finite number $n$ of particles. In practice we use a finite lattice and check whether a particle reaches the border. If a particle is about to reach the border we increase the size of the lattice. For a fixed value of $D$ we have simulated the system for several values of $n$, computing $\alpha$ by using (4). For each value of $D$, the critical value $\alpha_{c}$ was obtained in the limit $n \rightarrow \infty$ by a linear extrapolation in $1 / n$. Using these results we have built the phase diagram in the plane $D$ versus $\alpha$, as shown in Figs. 6, 7] and 8, The numerical values we have obtained for the transition line agrees very well with the results obtained previously for the ordinary models [7, 8].

When $D \rightarrow 1$ the critical value of $\alpha$ approaches a limiting value $\alpha_{0}$. For the singlecreation model $\alpha_{0}=1$ as expected from the exact result (66) and also from the mean-field result (9). Assuming that the behavior of $D$ around $\alpha=1$ is given by

$$
(1-D) \sim(1-\alpha)^{\phi}
$$

we have found from the plot shown in the inset of Fig. 6 that $\phi=4.03(3)$. Notice that the mean-field behavior, given by (9), predicts the value $\phi=1$.

An important feature of the models studied here is the emergence of a fractal structure at 


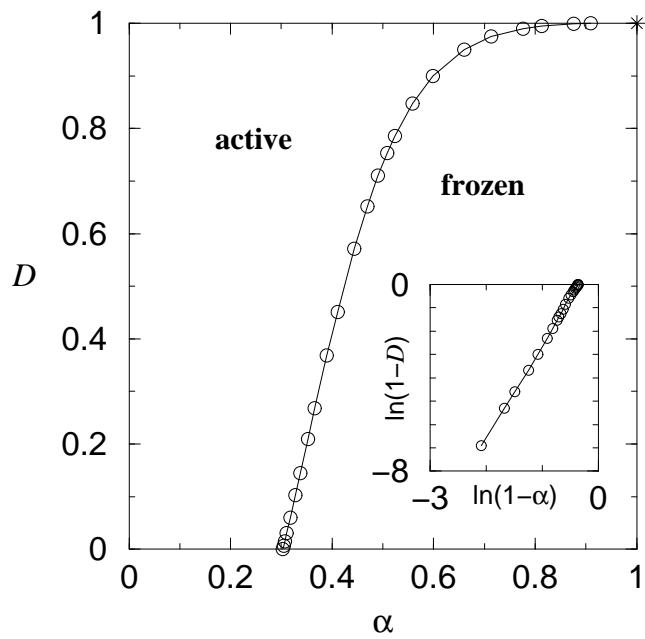

FIG. 6: Phase diagram for the single-creation conservative contact process. The star corresponds the value of $\alpha_{c}=1$ in the limit $D=1$. The inset corresponds the Log-log of equation (10). The transition from active to frozen state is always second-order.

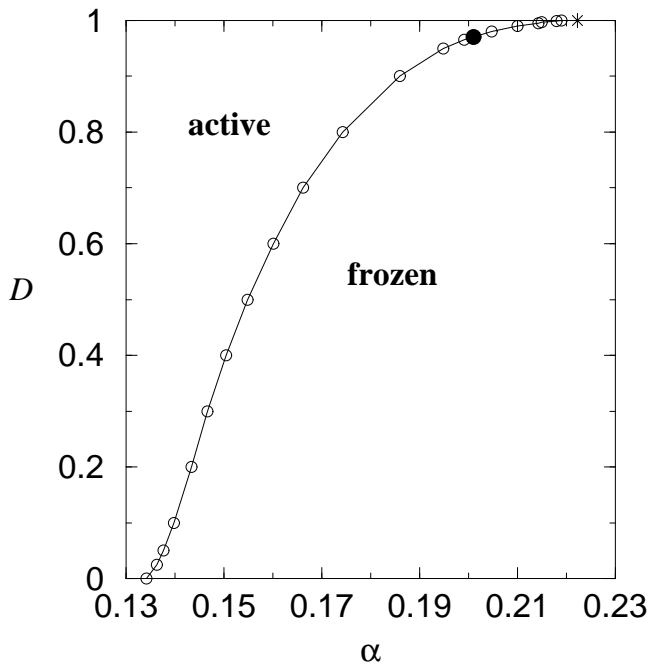

FIG. 7: Phase diagram for the pair-creation conservative contact process. The star corresponds the value of $\alpha_{0}=0.222$ in the limit $D=1$. The tricritical point (full circle) is located at $\alpha_{t}=0.199$ and $D_{t}=0.965$.

the transition point, characterized by its fractal dimension. We have calculated the fractal dimension at the transition for each value of $D$. To this end we have simulated a system with $n$ particles and determined the average distance $R$ between the two particles located 


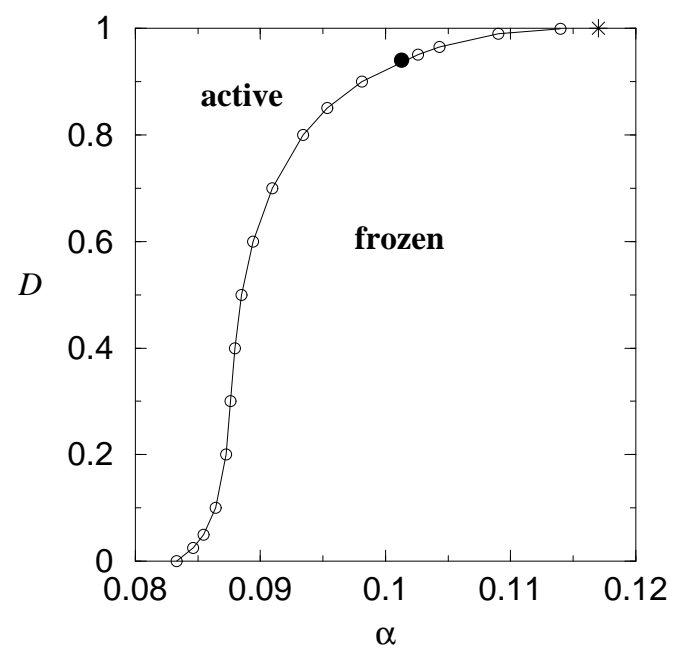

FIG. 8: Phase diagram for the triplet-creation conservative contact process. The star corresponds the value of $\alpha_{0}=0.115$ in the limit $D=1$ The tricritical point (full circle) is located at $\alpha_{t}=0.102$ and $D_{t}=0.945$.

at the extremities of the system. We assume the asymptotic behavior [10]

$$
n \sim R^{d_{F}}
$$

where $d_{F}$ is the fractal dimension, so that the slope of a $\log$-log plot of $N$ versus $R$ gives the fractal dimension as shown in Figs. 9] and 10, for the pair and triplet-creation models, respectively.

For a continuous phase transition studied here we expect the emergence of a fractal cluster with a fractal dimension $d_{F}$ strictly less than one. Indeed for the single-creation model and for the pair and triplet up to a certain value of $D$ we found a fractal dimension $d_{F}=0.75$ which is the expected value for a system in the DP universality class. However, for the pair and triplet creation and for sufficient large values of $D$, the fractal dimension becomes the Euclidean dimension $d_{F}=d=1$ reflecting the formation of a compact cluster whose size $R$ increases linearly with $n$. The changing of behavior occurs at $D_{t}=0.965 \pm 0.010$ and $\alpha_{t}=0.199 \pm 0.003$ for the pair-creation model and $D_{t}=0.945 \pm 0.005$ and $\alpha_{t}=0.102 \pm 0.001$ for the triplet-creation model. The tricritical point obtained by Dickman and Tomé [8] for the ordinary triplet-creation model by means of numerical simulations is $D_{t} \simeq 0.85$ and $\alpha_{t} \simeq 0.096$. These values correspond, actually, to a point over the critical line in Fig. 8 , For $D=0.85$ our result is $\alpha=0.0954$. As for the pair-creation model, the numerical results 


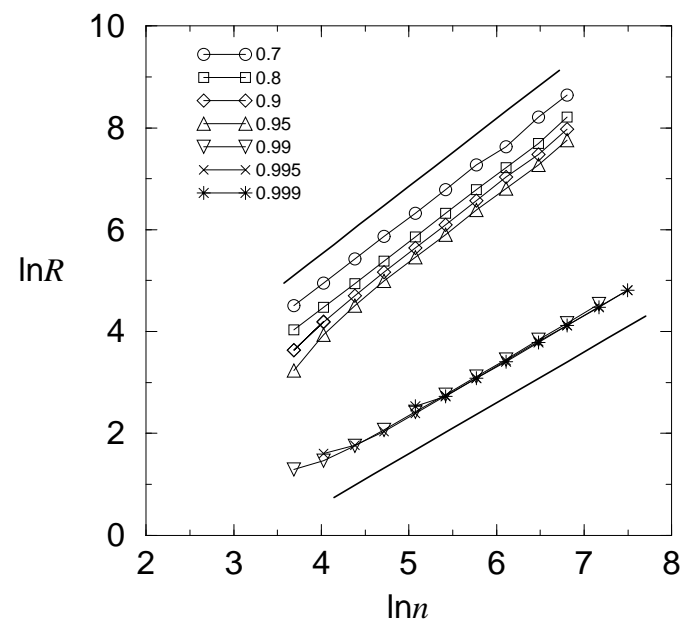

FIG. 9: Log-log plot of size of the system $R$, in the subcritical regime, as function of the number of particles $n$ for several values of probability $D$ for the pair-creation conservative contact process. The upper straight line has slope 1.33 and the lower one has slope 1.

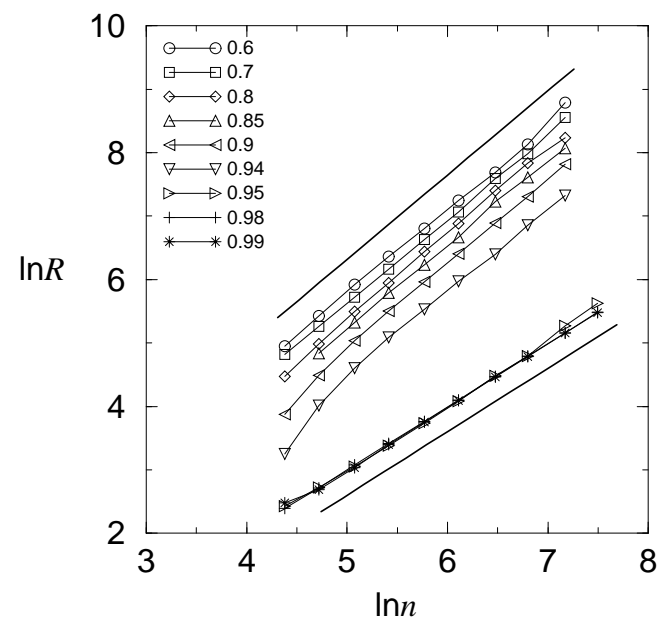

FIG. 10: Log-log plot of size of the system $R$, in the subcritical regime, as function of the number of particles $n$ for several values of probability $D$ for the triplet-creation conservative contact process. The upper straight line has slope 1.33 and the lower one has slope 1.

obtained by Dickman and Tomé [8] show that the transition is continuous for $D<0.95$ which is consistent with our results. However, they argue that the transition should remain continuous for any finite diffusion.

We argue that the formation of a compact cluster $\left(d_{F}=1\right)$ is a signature of a first order 
transition. First of all, the compact cluster has a nonzero density because $\rho=n / R$ does not vanish in the limit $n \rightarrow \infty$ and should therefore be identified with the active phase. Since the lattice is infinite the active phase is in coexistence with the frozen state (no particles). This behavior is very different from that corresponding to a continuous transition. In this case, the fractal dimension is less than one which cannot be identified with the active phase since the density $\rho=n / R \sim n^{-\left(1-d_{F}\right) / d_{F}} \rightarrow 0$ when $n \rightarrow \infty$.

When the cluster is a compact one, the ratio $n / R$ gives, in the limit $n \rightarrow \infty$, the density $\rho_{0}$ of the active phase in coexistence with the frozen phase. We have determined the values of $\rho_{0}$ for several values of $D$ above the tricritical point. An extrapolation for $D=1$ gives $\rho_{0}=0.665(1) \approx 2 / 3$ for the pair-creation model and $\rho_{0}=0.835(2) \approx 5 / 6$ for the tripletcreation model. The values of $\alpha_{0}$ at the first order transition can be obtained by substituting $\rho_{0}$ into the exact results (77) and (8). Using the numerical values, we get $\alpha_{0}=0.222(3) \approx 2 / 9$ and $\alpha_{0}=0.115(1) \approx 25 / 216$ for the pair-creation model, and the triplet-creation model, respectively. As stated before, these values are distinct from the spinodal values coming from the exact solutions (77) and (8). We remark, on the other hand, that the value of $\rho_{0}$ that we have obtained for the triplet-creation model agrees with the value $\rho=0.84$ obtained by Dickman and Tomé [8] for the active coexistence phase at $D=0.95$.

\section{CONCLUSION}

The effect of diffusion in nonequilibrium systems has been studied here for the case of three conservative contact processes. For the single-creation contact process, the diffusion does not change the nature of the phase transition, being continuous for any diffusion rate. This is expected since the usual contact process has already an intrinsic diffusion. Indeed, consider the following sequence of transitions $010 \rightarrow 011 \rightarrow 001$ starting with an isolated particle. The net result is a hopping of the isolated particle to a neighboring site, or effectively a diffusion. The sequence shown is a possible sequence of states for the single-creation which is carried out by a creation followed by a annihilation. This sequence, on the other hand, is not possible for the other two models.

For the pair-creation and and triplet-creation models the transition is continuous for low diffusion and becomes discontinuous for high enough diffusion. The present approach in which the number of particles is conserved is appropriate to study first order transition 
because it is possible to distinguish this transition from a continuous one by measuring the fractal dimension of the fractal cluster occurring at the critical point. If the fractal dimension is smaller than the dimension of the lattice the transition is continuous. When the cluster becomes compact, and the fractal dimension equals the lattice dimension, the transition becomes first order and, in addition, the density of particles turns out to be the density of the active phase in coexistence with the frozen phase.

\section{ACKNOWLEDGMENT}

C. E. F. thanks the financial support from Fundação de Amparo à Pesquisa do Estado de São Paulo (FAPESP) under Grant No. 03/01073-0.

[1] T. E. Harris, Ann. Probab. 2, 969 (1974).

[2] T. M. Ligget, Interacting Particle Systems (Springer-Verlag, New York, 1995).

[3] J. Marro and R. Dickman, Nonequilibrium Phase Transitions in Lattice Models (Cambridge University Press, Cambridge, 1999).

[4] R. Dickman, Phys. Rev. B 40, 7005 (1989).

[5] R. Dickman, J. Stat. Phys. 55, 997 (1989).

[6] R. Dickman and M. A. F. de Menezes, Phys. Rev. E 66045101 (2002) .

[7] I. Jensen and R. Dickman, J. Phys. A 26, L151 (1993).

[8] R. Dickman and T. Tomé, Phys. Rev. A 44, 4833 (1991).

[9] R. M. Ziff and B. J. Brosilow, Phys. Rev. A 46, 4630 (1992).

[10] H.-M. Bröker and P. Grassberger, Physica A 453 (1999).

[11] T. Tomé and M. J. de Oliveira, Phys. Rev. Lett. 86, 5643 (2001).

[12] H. J. Hilhorst and F. van Wijland, Phys. Rev. E 65, 035103 (2002).

[13] M. M. S. Sabag and M. J. de Oliveira, Phys. Rev. E 66, 036115 (2002).

[14] M. J. de Oliveira, Phys. Rev. E, 67 , 027104 (2003).

[15] R. Dickman, Phys. Rev. A 34, 4246 (1986).

[16] R. Dickman, Phys. Rev. A 42, 6985 (1990). 


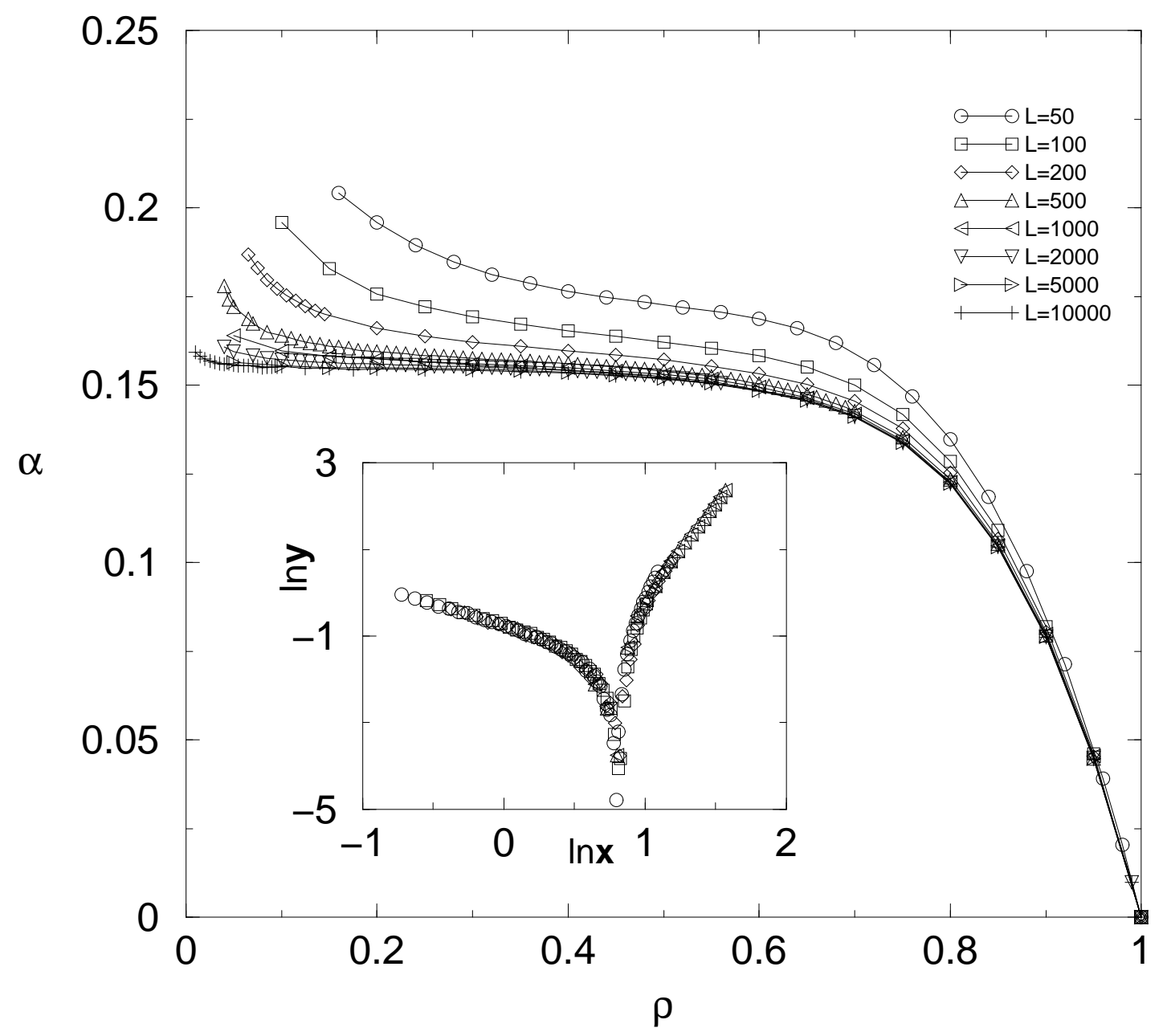

